\section{The role of $\alpha v$ integrins during angiogenesis: insights into potential mechanisms of action and clinical development}

\author{
Brian P. Eliceiri and David A. Cheresh \\ Departments of Immunology and Vascular Biology, IMM-24, The Scripps Research Institute, \\ 10550 North Torrey Pines Road, La Jolla, California 92037, USA. Phone: (619) 784-8281; \\ Fax: (619) 784-8926; E-mail: cheresh@scripps.edu.
}

Perspective

SERIES

Topics in angiogenesis

David A. Cheresh, Editor

This series continues

on pages 1231-1236 and

1237-1241 in this issue.
Angiogenesis depends on specific molecular interactions between vascular cells and components of the extracellular matrix (ECM). This Perspective focuses on the functional role of integrins in angiogenesis and neovascularization. Specifically, we discuss the mechanism by which antagonists of $\alpha v$ integrins disrupt angiogenesis in vivo and how they may impact patients with cancer and inflammatory disease.

Role of ECM and integrins during angiogenesis and vasculogenesis. Angiogenesis depends not only on growth factors and their receptors but is also influenced by receptors for ECM proteins. In general, cell adhesion to the ECM is mediated by integrins, heterodimeric transmembrane proteins that comprise a diverse family of over $15 \alpha$ and $8 \beta$ subunits. Integrin subunits can heterodimerize in over 20 combinations. Different integrin combinations may recognize a single ECM ligand, while others bind several different ECM proteins. Integrin-mediated adhesion leads to intracellular signaling events that regulate cell survival, proliferation, and migration (1). These signals include elevation in intracellular $\mathrm{pH}$ and calcium, inositol lipid synthesis, and the tyrosine phosphorylation of a wide range of nonreceptor tyrosine kinases such as focal adhesion kinase and Src kinases, as well as adaptor proteins such as Shc, p130 CAS, and Crk II. These signaling events trigger a number of downstream signals, including activation of the Ras/mitogen-activated protein (MAP) kinase pathway (1).

During angiogenesis, it is likely that a number of integrins expressed on the surface of activated endothelial cells regulate critical adhesive interactions with a variety of ECM proteins, including fibronectin, vitronectin, laminin, collagen types I and IV, von Willebrand factor, fibrinogen, and denatured collagen. Each of these adhesive interactions may regulate distinct biological events such as cell migration, proliferation, and differentiation. In addition, angiogenesis in different tissues may depend on specific endothelial cell interactions with ECMs that vary considerably in their adhesive protein composition.

Specific role of $\alpha$ vintegrins in angiogenesis. Of the wide spectrum of integrin subunit combinations that are expressed on the surface of cells, integrin $\alpha v \beta 3$ has been identified as having an especially interesting expression pattern among vascular cells during angiogenesis and vascular remodeling. Integrin $\alpha v \beta 3$ is a receptor for a wide variety of ECM ligands with an exposed RGD sequence, including vitronectin, fibronectin, fibrinogen, thrombospondin, proteolyzed collagen, von Willebrand factor, and osteopontin.

In the adult human, integrin $\alpha v \beta 3$ has a rather limited tissue distribution, as it is not typically expressed on epithelial cells and appears at minimal levels on intestinal, vascular, and uterine smooth muscle cells. This receptor is also expressed on a small percentage of activated leukocytes, macrophages, and osteoclasts, where it appears to contribute to immune function and bone resorption. Some invasive tumors, such as metastatic melanoma and late-stage glioblastoma, also express $\alpha v \beta 3$, where it contributes to malignant phenotype of the tumor (2). Endothelial cells exposed to growth factors, or those undergoing angiogenesis in tumors, wounds, or inflammatory tissue, express high levels of $\alpha v \beta 3$ (3-6). In fact, recent studies suggest that $\alpha v \beta 3$ may serve as a useful diagnostic or prognostic indicator of tumors. Specifically, the anti- $\alpha v \beta 3$ mAb LM609 coupled to a gadolinium-containing liposome specifically targeted tumor-associated blood vessels in rabbits, as detected by magnetic resonance imaging (7). Recent clinical studies indicate that radiolabeled humanized LM609 (Vitaxin) can be used to detect the vascular bed of human tumors and their metastatic lesions by wholebody imaging with a gamma camera (LoBoglio, A., and co-workers, unpublished observations).

The expression of $\alpha v \beta 3$ on activated endothelial cells suggests that this integrin may have an important function during angiogenesis and perhaps developmental neovascularization. In fact, disruption of $\alpha v \beta 3$ ligation with antibody (LM609) or peptide antagonists of $\alpha v \beta 3$ disrupts blood vessel formation in the chick chorioallantoic membrane (CAM), quail embryo, mouse retina, rabbit cornea, or arthritic knee $(4,6,8,9,10,11)$. These antagonists perturb the growth and/or maturation of new blood vessels without detectably influencing the preexisting blood vessels. In tumor models, inhibition of blood vessels by $\alpha v$ integrin antagonists not only blocked tumorassociated angiogenesis but in some cases caused tumor regression (5). Histological examination of tumors treated with the $\alpha v \beta 3$ antagonists revealed few, if any, viable tumor cells or detectable blood vessels (5).

Integrin $\alpha v \beta 3$ is capable of recognizing a number of ECM molecules in the context of the RGD adhesive sequence. However, recent studies suggest that $\alpha v \beta 3$ can bind the matrix metalloproteinase-2 (MMP-2) in a non-RGD-dependent manner where it serves to localize the active form of the enzyme on the surface of angiogenic blood vessels (12). This enables angiogenic endothelial cells to degrade and remodel the ECM during their invasion. Interestingly, native collagen contains RGD sites that are inaccessible to $\alpha v \beta 3$. However, after MMP-dependent proteolytic cleavage of collagen, these RGD sites are 


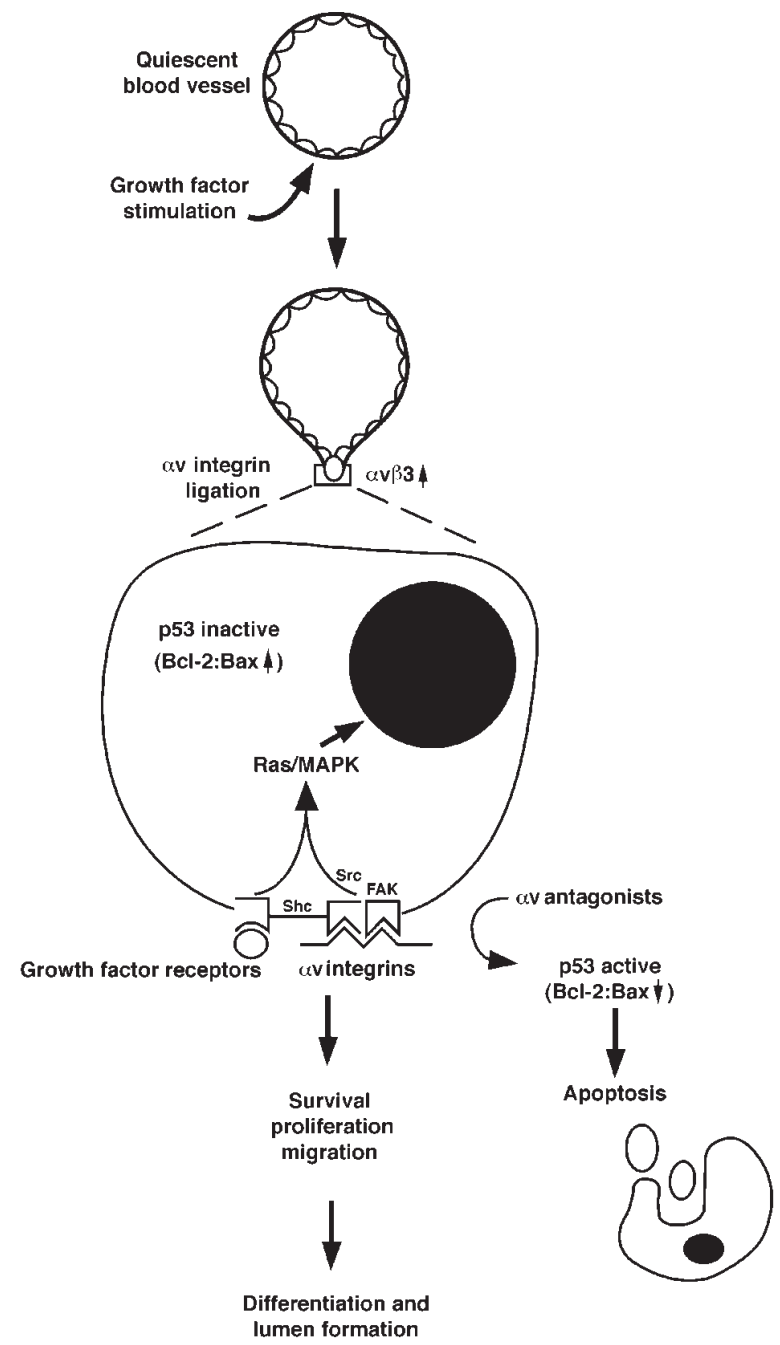

Figure 1

Hypothetical model for the role of $\alpha v$ integrins in angiogenesis. Angiogenic stimulus by growth factors induces expression of $\alpha v \beta 3$ and causes cells to invade the surrounding ECM and enter the cell cycle. When ligation of $\alpha v$ integrins is blocked, proliferating vascular cells undergo apoptosis, accompanied by an increase in p53 activity. Angiogenic growth factors induce the activation of Ras/MAP kinase in vascular cells and lead to cell proliferation, differentiation, and migration.

exposed and become ligated to $\alpha v \beta 3$. One might predict that the physical association between MMP- 2 and $\alpha v \beta 3$ might not only facilitate ECM remodeling but would enable $\alpha v \beta 3$-mediated endothelial cell invasion through the proteolyzed matrix by attachment to exposed RGD sites. In recent studies, natural breakdown fragments of MMP-2, termed PEX, were shown to accumulate in tissues that had undergone neovascularization (13). In fact, recombinant PEX was not only able to block MMP-2 binding to $\alpha v \beta 3$ but when administered in vivo was able to disrupt tumor-associated angiogenesis (13). Thus, PEX not only prevents endothelial cell-mediated matrix remodeling by MMP- 2 but would preclude $\alpha v \beta 3$ ligation by elimination of the exposed RGD sites in collagen.

Knockout mice reveal a direct role for $\alpha v$ integrins in vascularization. Recently, $\alpha_{v}$ integrin knockout mice have been developed to examine the role of the $\alpha v$ integrins in devel- opmental neovascularization (14). Mice deficient in $\alpha_{v}$ integrins express a dramatic vascular pattern. While most animals die in utero, approximately $20 \%$ of $\alpha \mathrm{v}$-deficient mice survive to term but die within hours after birth. This lethal mutation is associated with extensive brain and intestinal vessel abnormalities and hemorrhaging, demonstrating that $\alpha_{v}$ integrins are essential during blood vessel formation and/or maturation in these tissues. However, other organs show apparently normal vascularization (14), suggesting that animals lacking $\alpha v$ integrins can compensate to some degree in an organ-specific basis. Interestingly, mice lacking the $\beta 3$ subunit while having a bleeding disorder contain normal brain and intestinal blood vessels (15). Therefore, an objective comparison of the $\alpha \mathrm{v}$ and $\beta 3$ knockout mice would lead one to conclude that an $\alpha \mathrm{v}$ integrin other than $\alpha v \beta 3$ was required for brain and gastrointestinal tract neovascularization. The ability of some organs to support neovascularization, even in the absence of the $\alpha \mathrm{v}$ integrins, may provide a clue to some of the alternate integrins that may mediate vessel formation in the absence of $\alpha_{v} \beta 3$ or $\alpha v$ integrins. For example, integrin $\alpha v \beta 5$ can potentiate a pathway of angiogenesis distinct from that mediated by $\alpha v \beta 3$ (10). Moreover, mice lacking the fibronectin receptor $\alpha 5 \beta 1$ show embryonic lethality with multiple development problems, including severe vascular defects (16). In some cases, knockout mice have provided useful mechanistic information about a given molecule and its biological function. However, the lethality associated with a number of the integrin-deficient mice has made it difficult to decipher how these molecules function in normal animals. In addition, the likelihood for genetic compensation and redundancy provides an additional concern when interpreting the data from these models. Perhaps when conditional knockouts are studied we will gain further insight into the precise role of $\alpha_{v}$ and other integrins in vascular development.

Integrin $\alpha v \beta 3$ and vascular cell survival. The role of integrin $\alpha v \beta 3$ in mediating crucial steps of angiogenic blood vessels has led to the conclusion that this integrin facilitates the survival of stimulated endothelial cells. In fact, systemic administration of $\alpha v \beta 3$ antagonists to animals with ongoing angiogenesis shows blood vessels containing high levels of apoptotic endothelial cells. These results suggest that ligation of $\alpha v \beta 3$ on vascular cells may mediate a signaling event that is critical for the survival and differentiation of vascular cells undergoing angiogenesis in vivo. In this regard, $\alpha v \beta 3$ ligation is essential for the sustained activation of MAP kinase by angiogenic growth factors (17). Ligation of growth factor receptors and integrins by the appropriate ligands is known to potentiate a variety of intracellular signals, including the activation of the Ras/MAP kinase cascade and the activation of focal adhesion kinase (FAK) and the Src tyrosine kinases (Figure 1). In addition, $\alpha v \beta 3$ antagonists administered during angiogenesis in the chick CAM induce endothelial cell p53-dependent DNA-binding activity, which in turn leads to an increase in the expression of the cell cycle inhibitor $\mathrm{p} 21^{\mathrm{WAF} 1 / \mathrm{CIP} 1}$ and decreased expression of the death-promoting protein bax (18). Ligand binding by endothelial cell $\alpha v \beta 3$ suppresses this apoptotic pathway, thereby facilitating the ultimate differentiation of new blood vessels. It is important to point out that mice 
clearly survive without $\mathrm{p} 53$, indicating that alternative or compensatory apoptotic pathways can allow normal development. The importance of p53 during $\alpha \mathrm{v}$-mediated angiogenesis and endothelial cell survival is underscored by recent preliminary evidence that p53-deficient mice are refractory to the effects of $\alpha \mathrm{v}$ antagonists (Stromblad, R., et al., unpublished observations). This provides strong evidence that endothelial cell survival through p53 activity is linked to $\alpha_{\mathrm{v}}$ binding to the ECM. In the absence of $\mathrm{p} 53$, another compensatory pathway is engaged, facilitating endothelial cell survival in an $\alpha_{v-}$ independent manner. These observations may help explain the compensatory mechanism facilitating blood vessel growth in $\alpha v$-deficient mice.

Two angiogenic pathways are characterized by distinct $\alpha v$ integrins. Two pathways of angiogenesis have recently been identified based on their dependence on the related but distinct integrins $\alpha_{v} \beta 3$ and $\alpha_{v} \beta 5$ (10). In both the rabbit corneal eye pocket and the chick chorioallantoic membrane angiogenesis assays, anti- $\alpha \mathrm{v} \beta 3 \mathrm{mAb}$ blocked bFGF-induced angiogenesis, whereas anti- $\alpha v \beta 5$ antagonists blocked VEGF-induced angiogenesis. Furthermore, inhibition of the protein kinase $C$ pathway blocked the VEGF-induced angiogenesis and did not affect bFGF-induced angiogenesis. The biological relevance of these distinct angiogenic pathways is unknown. Perhaps, vascular remodeling in distinct organs depends on the particular growth factors and/or adhesive proteins contained within the specific ECM. As stated above, $\beta 3$ integrin-deficient mice have normal brain and gut vessel development in the absence of $\alpha_{v} \beta 3$, while $\alpha_{v}$ integrin-deficient mice have defective brain and gut blood vessels. This strongly implicates another $\alpha_{v}$ integrin, perhaps $\alpha v \beta 5$, in brain and gut developmental neovascularization.

Clinical perspectives. Based on the strong preclinical data in animal models, the clinical potential of $\alpha_{\mathrm{V}}$-integrin antagonists is currently being evaluated in phase I and phase II clinical trials for patients with late-stage cancer. For example, the humanized form of the anti- $\alpha v \beta 3 \mathrm{mAb}$ LM609 (Vitaxin) has successfully completed phase I trials. Of the 14 cancer patients evaluated, 8 showed disease stabilization and/or some objective tumor reduction (Table 1). Importantly, there was no evidence of toxicity at all doses tested, even when administered for 22 consecutive months. This clinical trial is among the first use of a "targeted" antiangiogenic approach in humans. These clinical data closely mirror the effects observed in tumor-bearing animal models (5). Phase II cancer trials are now under way to further evaluate the efficacy of Vitaxin during longterm treatment. Additional phase I trials are under way to assess the effects of an $\alpha v \beta 3 / \alpha v \beta 5$ small-molecule antagonist in late-stage cancer patients.

Conclusions. Further study of the basic cell biological mechanisms underlying growth factor receptors and integrin function during angiogenesis may continue to provide insight into the evaluation of the clinical bene-

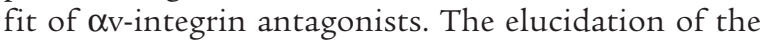
molecular basis of angiogenesis remains a challenge because of the complex interactions between the ECM and vascular cells that must be temporally and spatially coordinated. Examination of the signaling events trans-

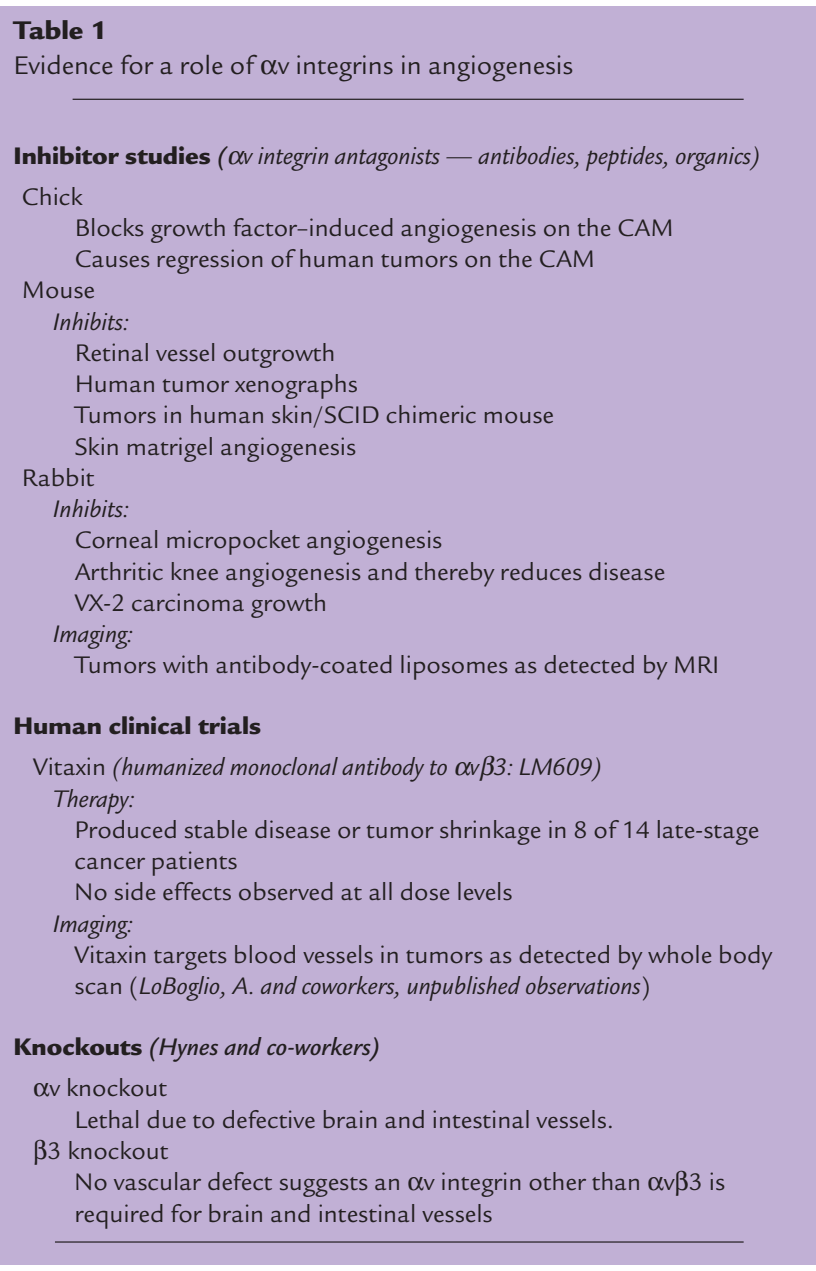

duced by cell adhesion molecules to the smooth muscle and endothelial cells may reveal mechanisms in which cells can process cytokine or growth factor stimuli to impact changes in intracellular phosphorylation cascades, gene expression levels, and ECM-associated enzymatic activities. The coordinated response to these inputs will likely impact the processes of vascular cell survival, invasion, migration, proliferation, and differentiation during angiogenesis. Knowledge of these molecular mechanisms will foster the development of novel antiangiogenic therapies to benefit patients with cancer and inflammatory disease.

\section{Acknowledgments}

B.P. Eliceiri was supported by a National Research Service Award postdoctoral fellowship(1F32 HL-09435). D.A. Cheresh was supported by grants CA-50286, CA-45726, CA-78045, and HL54444 from the National Institutes of Health.

\footnotetext{
1. Aplin, A.E., Howe, A., Alahari, S.K., and Juliano, R.L. 1998. Signal transduction and signal modulation by cell adhesion receptors: the role of integrins, cadherins, immunoglobulin-cell adhesion molecules, and selectins. Pharmacol. Rev. 50:197-263.

2. Felding-Habermann, B., Mueller, B.M., Romerdahl, C.A., and Cheresh, D.A. 1992. Involvement of integrin $\alpha_{\mathrm{v}}$ gene expression in human melanoma tumorigenicity. J. Clin. Invest. 89:2018-2022.

3. Enenstein, J., and Kramer, R.H. 1994. Confocal microscopic analysis of integrin expression on the microvasculature and its sprouts in the neonatal foreskin. J. Invest. Dermatol. 103:381-386.

4. Brooks, P.C., Clark, R.A., and Cheresh, D.A. 1994. Requirement of vascular integrin $\alpha v \beta 3$ for angiogenesis. Science. 264:569-571.
} 
5. Brooks, P.C., et al. 1994. Integrin $\alpha v \beta 3$ antagonists promote tumor regression by inducing apoptosis of angiogenic blood vessels. Cell. 79:1157-1164.

6. Storgard, C.M., et al. 1999. Decreased angiogenesis and arthritic disease in rabbits treated with an $\alpha v \beta 3$ antagonist. J. Clin. Invest. 103:47-54.

7. Sipkins, D.A., et al. 1998. Detection of tumor angiogenesis in vivo by $\alpha v \beta 3$-targeted magnetic resonance imaging. Nat. Med. 4:623-626.

8. Drake, C.J., Cheresh, D.A., and Little, C.D. 1995. An antagonist of integrin $\alpha v \beta 3$ prevents maturation of blood vessels during embryonic neovascularization. J. Cell Sci. 108:2655-2661.

9. Brooks, P.C., et al. 1995. Antiintegrin $\alpha v \beta 3$ blocks human breast cancer growth and angiogenesis in human skin. J. Clin. Invest. 96:1815-1822.

10. Friedlander, M., et al. 1995. Definition of two angiogenic pathways by distinct $\alpha_{\mathrm{V}}$ integrins. Science. 270:1500-1502.

11. Hammes, H.P., Brownlee, M., Jonczyk, A., Sutter, A., and Preissner, K.T. 1996. Subcutaneous injection of a cyclic peptide antagonist of vitronectin receptor-type integrins inhibits retinal neovascularization. Nat. Med. 2:529-533.

12. Brooks, P.C., et al. 1996. Localization of matrix metalloproteinase MMP2 to the surface of invasive cells by interaction with integrin $\alpha v \beta 3$. Cell.
85:683-693.

13. Brooks, P.C., Silletti, S., von Schalscha, T.L., Friedlander, M., and Cheresh, D.A. 1998. Disruption of angiogenesis by PEX, a noncatalytic metalloproteinase fragment with integrin binding activity. Cell. 92:391-400.

14. Bader, B.L., Rayburn, H., Crowley, D., and Hynes, R.O. 1998. Extensive vasculogenesis, angiogenesis, and organogenesis precede lethality in mice lacking all alpha v integrins. Cell. 95:507-519.

15. Hodivala-Dilke, K.M., et al. 1999. $\beta 3$-integrin-deficient mice are a model for Glanzmann thrombasthenia showing placental defects and reduced survival. J. Clin. Invest. 103:229-238.

16. Yang, J.T., Rayburn, H., and Hynes, R.O. 1993. Embryonic mesodermal defects in $\alpha 5$ integrin-deficient mice. Development. 119:1093-1105.

17. Eliceiri, B.P., Klemke, R., Stromblad, S., and Cheresh, D.A. 1998. Integrin $\alpha v \beta 3$ requirement for sustained mitogen-activated protein kinase activity during angiogenesis. J. Cell Biol. 140:1255-1263.

18. Stromblad, S., Becker, J.C., Yebra, M., Brooks, P.C., and Cheresh, D.A. 1996. Suppression of $\mathrm{p} 53$ activity and $\mathrm{P} 21 \mathrm{WAF} 1 / \mathrm{CIP} 1$ expression by vascular cell integrin $\alpha_{\mathrm{v}} \beta 3$ during angiogenesis. J. Clin. Invest. 98:426-433. 